Case Report

\title{
Rare Cause of Late Recurrent Angina following Coronary Artery Bypass Grafting: Iatrogenic Aortocoronary Arteriovenous Fistula Causing Coronary Steal
}

\author{
Jayakumar Sreenivasan $\mathbb{D D}^{1}{ }^{1}$ Muhammad Ayub, ${ }^{1}$ Neha Yadav, ${ }^{2}$ and Yasmeen Golzar ${ }^{2}$ \\ ${ }^{1}$ Department of Internal Medicine, John H. Stroger Hospital of Cook County, Chicago, IL 60612, USA \\ ${ }^{2}$ Department of Internal Medicine, Division of Cardiology, John H. Stroger Hospital of Cook County, Chicago, IL 60612, USA \\ Correspondence should be addressed to Jayakumar Sreenivasan; jayakumars101@gmail.com
}

Received 10 February 2018; Revised 19 April 2018; Accepted 2 May 2018; Published 24 June 2018

Academic Editor: Hajime Kataoka

Copyright (c) 2018 Jayakumar Sreenivasan et al. This is an open access article distributed under the Creative Commons Attribution License, which permits unrestricted use, distribution, and reproduction in any medium, provided the original work is properly cited.

Iatrogenic aortocoronary arteriovenous fistula is a very rare complication of coronary artery bypass grafting in which one of the arterial grafts inadvertently forms a fistulous tract with a cardiac vein, shunting blood from the anastomosed coronary artery. We report a patient with an iatrogenic left internal mammary artery graft to cardiac vein fistula presenting with recurrent angina three years after a three-vessel coronary artery bypass grafting.

\section{Introduction}

This case of iatrogenic aortocoronary arteriovenous fistula (ACAVF) is interesting because it is a rare but important complication of coronary artery bypass grafting (CABG) which can result in significant clinical consequences like heart failure, tamponade, and death. To the best of our knowledge, only 38 similar cases of ACAVF have been reported in the literature. We have included a brief review of the literature on this rare complication and the various available treatment options.

\section{Case Presentation}

A 61-year-old woman with diabetes mellitus, hypertension, and hyperlipidemia initially presented with unstable angina; a regadenoson stress nuclear myocardial perfusion imaging (MPI) revealed anterolateral wall ischemia. Subsequent coronary angiography demonstrated severe stenoses of the left anterior descending (LAD) artery, left circumflex artery, and right coronary artery. She underwent CABG with a left internal mammary artery (LIMA) graft to the LAD artery and saphenous venous grafts (SVG) to the right posterior descending (RPDA) and obtuse marginal 4 (OM4) arteries.
After CABG, the patient was doing well on guidelinedirected medical therapy. Three years later, she presented with acute onset of chest pain suggestive of unstable angina. On admission, her heart rate was $80 / \mathrm{min}$, blood pressure was 140/86 mmHg, and her physical exam was unremarkable.

\section{Investigations}

Electrocardiogram showed normal sinus rhythm without any evidence of ischemia. Serially monitored cardiac enzymes were within normal limits, and transthoracic echocardiography revealed normal ejection fraction with no distinct regional wall motion abnormalities. Regadenoson stress nuclear MPI showed anterolateral ischemia, a territory which should have been supplied by the LIMA to LAD graft. Diagnostic coronary angiography revealed known severe native, three-vessel coronary artery disease. The native LAD vessel was very small. Proximal LAD was medium sized. Mid LAD had $100 \%$ stenosis at the origin of diagonal branch. Distal LAD was not visualized (Figure 1 and Video 1). Graft angiography was significant for a very large tortuous LIMA graft with poor visualization of the LAD artery after touchdown (Figure 2 and Video 2). Upon contrast injection of the LIMA graft, there was immediate opacification of the 


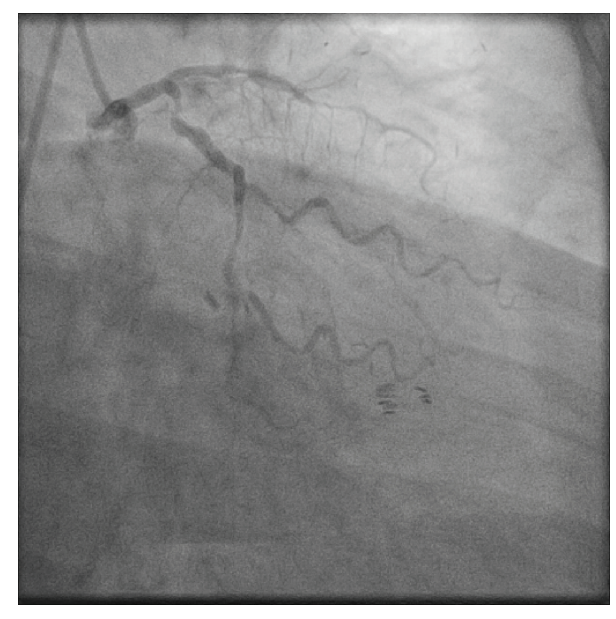

FIGURE 1: Right anterior oblique (RAO) caudal view of the left coronary artery with severe disease of the left anterior descending (LAD) artery.

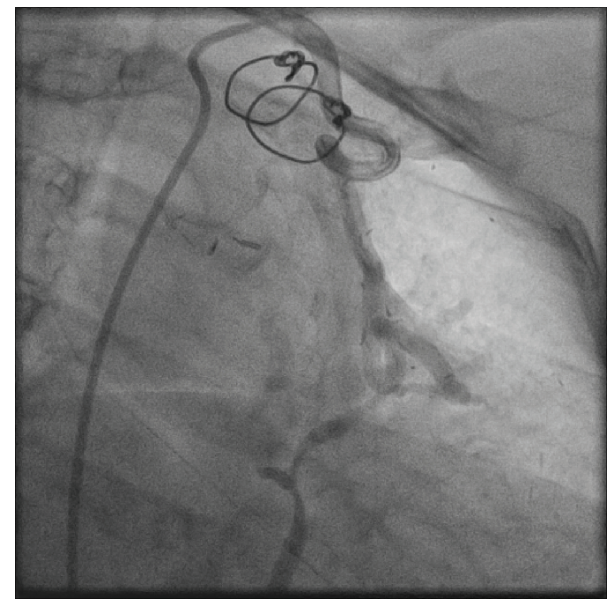

FIGURE 2: Graft angiography demonstrated a tortuous left internal mammary artery (LIMA) graft with flow going to the coronary sinus forming an aortocoronary arteriovenous fistula.

coronary sinus suggesting a fistulous tract between the LIMA graft and a cardiac vein (Figure 2 and Video 1). SVG grafts to the RPDA and OM4 arteries were patent. Cardiac computed tomographic angiography (CTA) confirmed the cardiac catheterization findings of a LIMA graft to coronary sinus fistula (Figure 3).

\section{Clinical Decision Making, Treatment, Outcome, and Follow-Up}

The case was discussed with the cardiac surgical team as well as in a multicenter interventional conference to explore both surgical and percutaneous options. A percutaneous approach of coiling of the fistula is anatomically complex; a surgical approach which would involve ligation of ACAVF and redo CABG, would put the patient at higher risk of perioperative cardiac morbidity and mortality. Because of the coronary arterio-venous fistula with steal, it was unclear whether

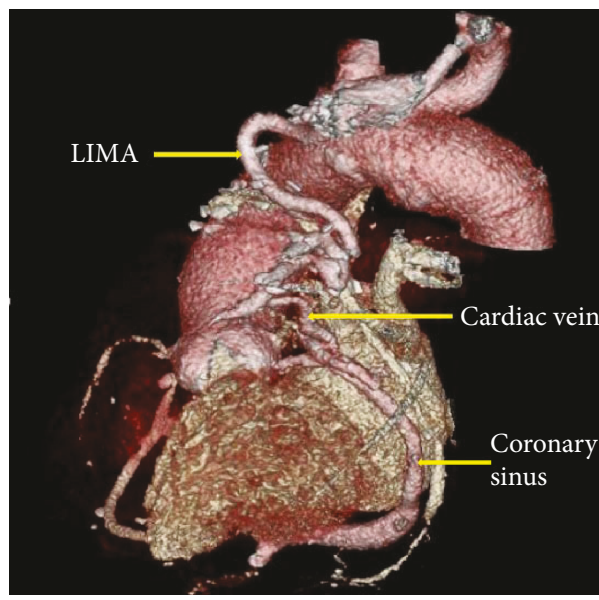

FIgURE 3: Cardiac computed tomography (CT) angiography with 3dimensional reconstruction showed distal left internal mammary artery (LIMA) to left anterior descending (LAD) bypass artery communicating with a cardiac vein, which is eventually communicating with the coronary sinus, suggesting a coronary arteriovenous fistula.

revascularization of the native LAD would be of any significant benefit to the patient. After full discussion about the risks versus benefits of treatment options with the heart team, the patient elected for medical management and intervention only if she has escalating symptoms. The patient's antianginal therapy was optimized with Metoprolol $50 \mathrm{mg}$ daily, which she tolerated well with gradual improvement in her exercise tolerance and resolution of her anginal symptoms. The patient is currently asymptomatic at 1 year follow-up. Repeat cardiac stress testing may be pursued if she has recurrent symptoms. The tentative plan is to offer her interventional coil closure or surgical closure of the ACAVF if she has recurrent angina or heart failure symptoms.

\section{Discussion}

Iatrogenic ACAVF is a rare complication of CABG causing significant morbidity. To the best of our knowledge, only 38 cases have been reported [1]. ACAVF can become symptomatic over the course of time. The most common presentation is angina due to coronary steal phenomenon involving the affected coronary artery [2]. Other less commonly reported potential outcomes are high output heart failure as a sequela of chronic arteriovenous fistula and rupture leading to cardiac tamponade [2-4]. The LAD is the most commonly involved artery, representing $60 \%$ of cases of iatrogenic ACAVF following CABG [2]. Possible risk factors for development of post-CABG iatrogenic ACAVF are myocardial fibrosis from prior infarction or cardiac surgery as well as a LAD artery deeply embedded in thick epicardial fat which makes it surgically challenging to separate it from the adjacent great cardiac vein $[2,3]$. Cardiac computed tomography (CT) or magnetic resonance angiography (MRA) are the best available noninvasive imaging modalities for visualization of ACAVF [5, 6]. Cardiac catheterization with graft angiography is the best testing modality for the diagnosis of ACAVF. The appearance on angiography is a very large caliber LIMA 
which has undergone hypermaturation due to it being connected to a low-pressure venous system. As of now, there are no robust evidence-based data to help guide treatment for patients with ACAVF. Management of ACAVF depends on the presentation and symptomatology of the patients. Spontaneous closure of ACAVF in two asymptomatic patients has been reported so far [7]. Hence, conservative therapy is preferred for asymptomatic patients and patients with a good response to optimal medical treatment of angina as like in our case [2]. Our case is the third in literature for conservative medical management of ACAVF. We think medical management of ACAVF should include antianginal therapy particularly, a beta blocker in line with medical therapy for stable coronary artery disease until we have further prospective investigations for this unique clinical scenario. Patients who are persistently symptomatic due to ACAVF despite optimal medical therapy have primarily been treated with surgical ligation of the fistula and bypassing the diseased coronary artery [3]. With current advancements in innovative percutaneous techniques, coil or balloon embolization of the fistula and stenting of the unbypassed artery are the promising alternative treatment options over surgical approaches [8-13].

\section{Consent}

Written explicit consent was obtained from the patient by the corresponding author for reporting and publication of all case details including imaging and follow-up data. Consent forms are available if requested by editorial team.

\section{Disclosure}

Abstract of the case was presented as a challenging case in Transcatheter Cardiovascular Therapeutics (TCT) 2017 Annual Conference held in Denver, CO.

\section{Conflicts of Interest}

The authors declare that they have no conflicts of interest.

\section{Supplementary Materials}

Supplementary 1. Video 1: left coronary angiography showing medium-sized proximal LAD with $100 \%$ stenosis at the origin of the first diagonal branch and poorly visualized distal LAD. First diagonal and left circumflex artery had severe diffuse atherosclerosis.

Supplementary 2. Video 2: graft angiography revealing a very large-sized, tortuous left internal mammary artery (LIMA) graft with flow going to the coronary sinus from a coronary arteriovenous fistula and poor visualization of left anterior descending (LAD) artery after touchdown.

\section{References}

[1] E. Ornek, H. Kundi, E. Kiziltunc, and M. Cetin, "Treatment of iatrogenic aortocoronary arteriovenous fistula with coronary covered stent," Case Reports in Cardiology, vol. 2016, Article ID 9126817, 3 pages, 2016.

[2] J. D. Gardner, W. R. Maddox, and J. B. Calkins, "Iatrogenic aortocoronary arteriovenous fistula following coronary artery bypass surgery: a case report and complete review of the literature," Case Reports in Cardiology, vol. 2012, Article ID 652086, 9 pages, 2012.

[3] J. B. Calkins Jr, J. D. Talley, and N. H. Kim, "Iatrogenic aortocoronary venous fistula as a complication of coronary artery bypass surgery: patient report and review of the literature," Catheterization and Cardiovascular Diagnosis, vol. 37, no. 1, pp. 55-59, 1996.

[4] C. Khunnawat, S. Mukerji, G. S. Abela, and R. K. Thakur, "Unusual complications of coronary artery bypass graft surgery," The American Journal of Cardiology, vol. 98, no. 12, pp. 1665-1666, 2006.

[5] J. D. Schilling and J. J. Billadello, "Right coronary artery to coronary sinus fistula by transesophageal echocardiogram, cardiac magnetic resonance imaging, and coronary angiography," Clinical Cardiology, vol. 32, no. 7, pp. E29E30, 2009.

[6] H. J. Seon, Y. H. Kim, S. Choi, and K. H. Kim, "Complex coronary artery fistulas in adults: evaluation with multidetector computed tomography," The International Journal of Cardiovascular Imaging, vol. 26, Supplement 2, pp. 261-271, 2010.

[7] U. Deligonul, M. Vandormael, J. M. Harper, and M. J. Kern, "Spontaneous closure of iatrogenic left internal mammary artery to anterior interventricular vein fistula," The American Journal of Cardiology, vol. 58, no. 9, pp. 854-855, 1986.

[8] P. Braun, R. Höltgen, E. Stroh et al., "Coil embolization of an AV-fistula between the left thoracic artery and vein after coronary artery bypass surgery," Zeitschrift für Kardiologie, vol. 88, no. 10, pp. 812-814, 1999.

[9] I. S. Jung, J. O. Jeong, S. S. Kim et al., "Iatrogenic left internal mammary artery to great cardiac vein anastomosis treated with coil embolization," Korean Circulation Journal, vol. 41, no. 2, pp. 105-108, 2011.

[10] L. S. Maier, A. B. Buchwald, B. Ehlers, K. Rühmkorf, and K. H. Scholz, "Closure of an iatrogenic aortocoronary arteriovenous fistula: transcatheter balloon embolization following failed coil embolization and salvage of coils that migrated into the coronary venous system," Catheterization and Cardiovascular Interventions, vol. 55, no. 1, pp. 109-112, 2002.

[11] A. A. Miranda, J. A. Hill, J. P. Mickle, and R. G. Quisling, "Balloon occlusion of an internal mammary artery to anterior interventricular vein fistula," The American Journal of Cardiology, vol. 65, no. 3, pp. 257-258, 1990.

[12] I. Sheiban, C. Moretti, and S. Colangelo, "Iatrogenic left internal mammary artery-coronary vein anastomosis treated with covered stent deployment via retrograde percutaneous coronary sinus approach," Catheterization and Cardiovascular Interventions, vol. 68, no. 5, pp. 704-707, 2006.

[13] W. J. Thomas, W. B. Moskowitz, A. Freedman, G. W. Vetrovec, and E. Goudreau, "Therapeutic embolization for unusual latrogenic complications related to coronary revascularization," Catheterization and Cardiovascular Interventions, vol. 46, no. 4, pp. 457-462, 1999. 


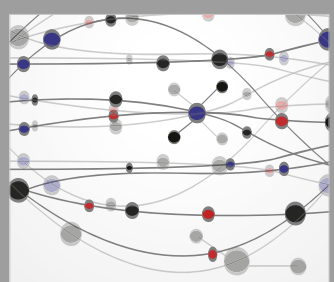

The Scientific World Journal
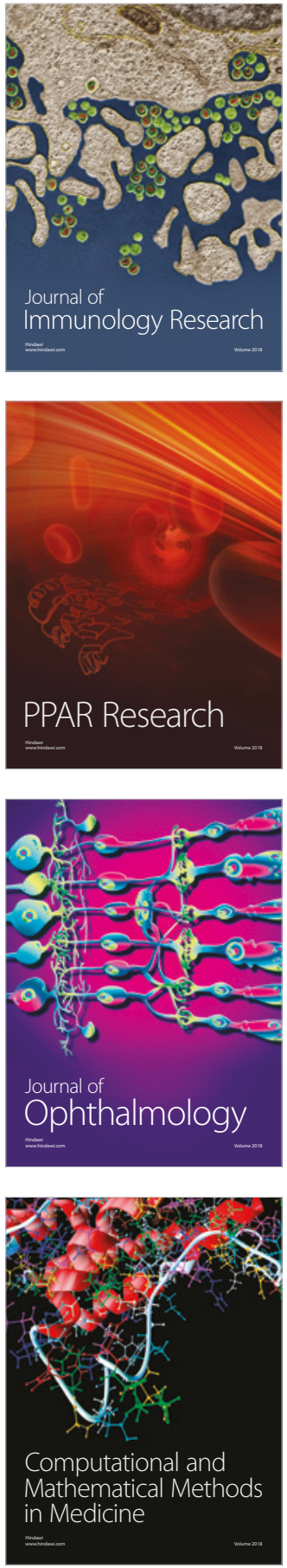

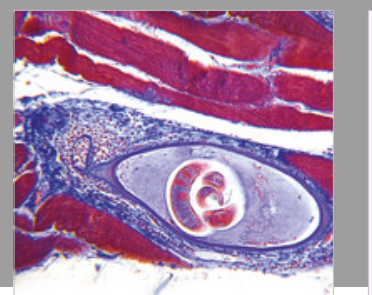

Gastroenterology Research and Practice

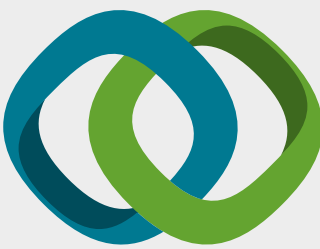

\section{Hindawi}

Submit your manuscripts at

www.hindawi.com
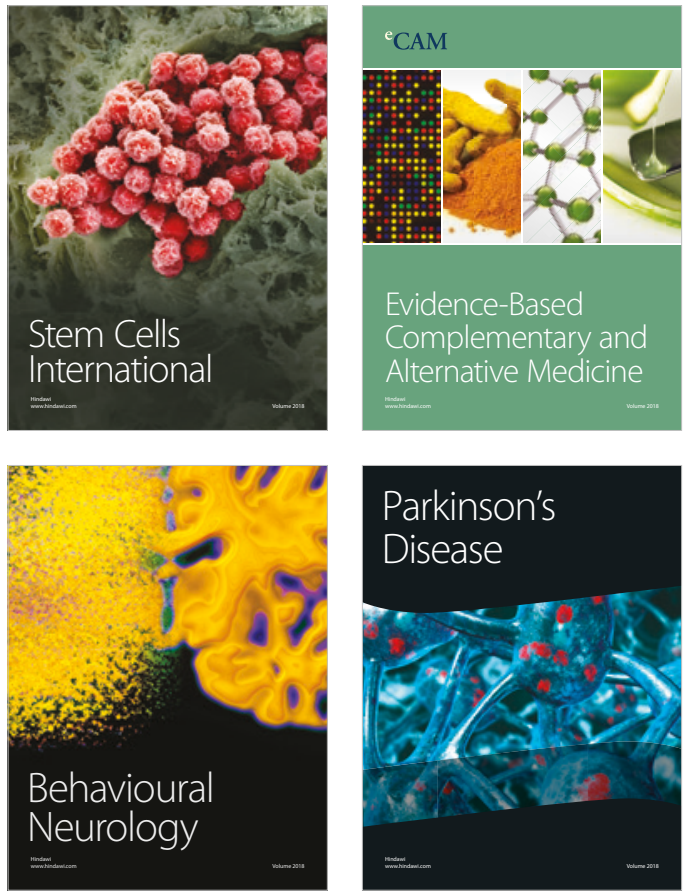

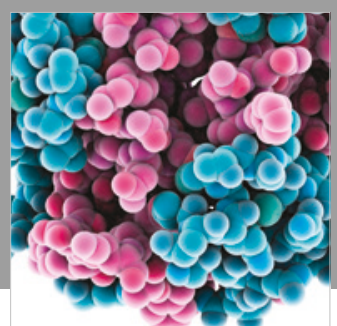

ournal of

Diabetes Research

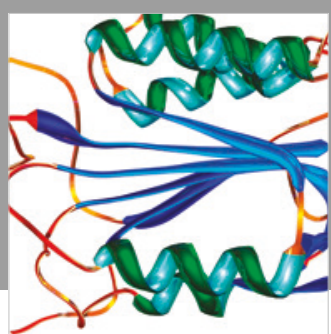

Disease Markers
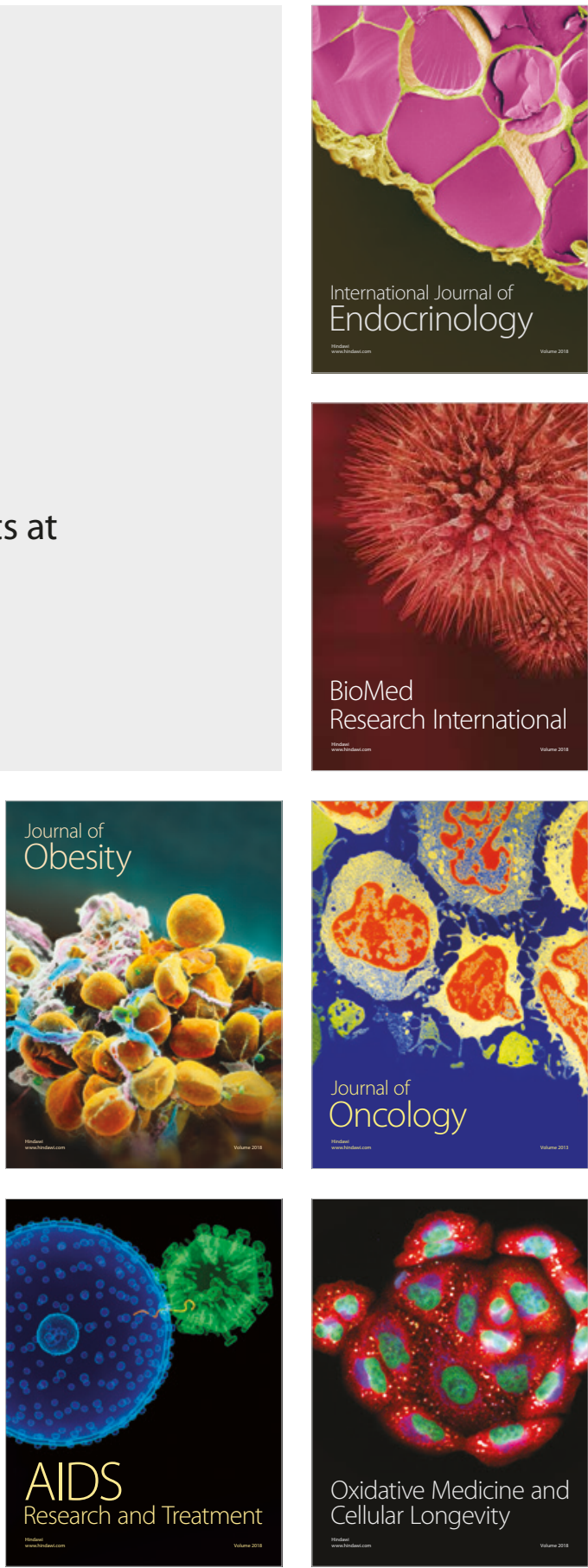